\title{
RESPUESTA AL THATCHERISMO EN TOP GIRLS Y SERIOUS MONEY DE CARYL CHURCHILL
}

\author{
Eva María Cortés Martínez . Universidad de Almería
}

\begin{abstract}
El propósito de este artículo es estudiar la respuesta de Caryl Churchill ${ }^{1}$ al Thatcherismo en Top Girls (1982) y Serious Money (1987), obras que describen la situación de Inglaterra en la década de los ochenta. Serious Money es una crítica de la avaricia y la corrupción presentes en los hombres de negocios en esta época, mientras que en Top Girls podemos ver una crítica feminista socialista al feminismo burgués. Por tanto, Churchill nos presenta una sociedad que necesita un cambio.
\end{abstract}

Este artículo estudia la respuesta de Caryl Churchill a las medidas económicas y políticas de Margaret Thatcher, en sus obras Serious Money y Top Girls, donde se analiza la política conservadora del Thatcherismo.

En la década de los ochenta en Inglaterra, Margaret Thatcher- conocida como "la Dama de Hierro"- llevó a cabo una profunda reestructuración del Estado mediante medidas de carácter político, social y económico. Dichas medidas se basaban en un individualismo feroz, cuya doctrina consistía básicamente en el denominado "capitalismo popular", desarrollado en una sociedad jerarquizada y dominada por el hombre. Como O’Shaughnessy (1996:296) señala, el Thatcherismo definido como "authoritarian populism"consiste en "he linkage of popular themes and discontents (law and order, immigration, education standards, the undermining of family values) with a thoroughgoing critique of social democracy in a way that justifies the reinforcement of the state and the 'freeing' of the market".

Anteriormente existía una actitud de consenso entre las fuerzas políticas que ya desde principios de los años sesenta estaba pasando por una etapa crítica, viéndose fragmentada con mayor intensidad con la llegada de Margaret Thatcher al poder tras ganar las elecciones el tres de mayo de 1979, iniciándose así la etapa thatcherista vigente durante los ochenta.

\footnotetext{
${ }^{1}$ Nacida en Londres, el 3 de septiembre de 1938, Caryl Churchill es considerada como una de las más distinguidas dramaturgas del teatro inglés contemporáneo. Sus obras son crudas, muy francas y agudas en su crítica a las instituciones sociales. Ella escribe un teatro alternativo, pero que es altamente comercial. En sus obras se pueden ver sus intereses feministas y una política socialista.
} 
Las medidas económicas empleadas supusieron desde el principio una ruptura con todo lo visto anteriormente, debido en parte a que dichas medidas consistían en decisiones tomadas como consecuencia de la filosofía individualista de la Primera Ministra. Entre las medidas económicas adoptadas habría que resaltar el énfasis en regular la inflación (olvidando por completo el problema del desempleo), el sistema de leyes que reducía el poder de los sindicatos, un amplio programa de privatizaciones y el ya mencionado capitalismo popular.

Pero el Thatcherismo no sólo consistía en un programa basado en medidas económicas, sino que también constituía un sistema de valores morales que

se caracteriza por una vuelta al modelo victoriano de familia; la confianza en virtudes tales como el ahorro y la austeridad; la aversión al despilfarro y a la obstentación, y la fe en un nuevo credo, el individualismo y su precepto de autoayuda, prioritario al de la solidaridad y complementario a la intención del gobierno de romper la dependencia del individuo con respecto del estado en todos sus niveles... (Fernández Sánchez, 1999:14)

Se podría decir que parte del éxito de la política thatcherista se debía a la manera en la que elaboró un discurso propio. Margaret Thatcher se propuso reescribir el lenguaje de los politicos y desacreditar así las voces de los grupos sociales oponentes. Entre las técnicas empleadas, podemos mencionar su famosa metáfora de la búsqueda de la "verdad". Con este recurso, Thatcher concebía la política en términos de oposiciones binarias (bueno/ malo; verdadero/falso; esperanza/desesperación). A este respecto O'Shaughnessy (1996: 295-296) comenta que

The vision proposed is manichean - of polar and irreconcilable opposites rather than minor differences (...) the speaker creates for hersef the role of prophet - the bringer of truth and faith. She promises not only political change, but also a radical renaming of what has gone before. She also implicitly proposes a narrative model which she will be able to use to recount her years in office - that of the battle of the crusader for truth and hope against the forces of error and despair.

Una vez visto brevemente en qué consistía la política económica, los valores morales y el uso del lenguaje propios del Thatcherismo, vamos a ver la respuesta de Caryl Churchill en Serious Money y Top Girls .

Diversas opiniones se han vertido acerca de esta respuesta. Michel Wandor (en Thomas, 1992:160) dice que las obras de Churchill "display an equivocal attitude to change". De igual modo, Helene Keysar (Ibíd.) habla de "the absence of any positive strategy to change the dismal enslavement of women". Para Jane Thomas (1992:162) el método de Churchill "is not concerned with providing answers but with asking questions.It does not present a series of programmes for social reform but various analyses of power and the ways in which men govern (themselves and others) by the production of truth."

Por nuestra parte, este artículo quiere demostrar que la postura de Churchill no es ambigua y, por tanto, lo que vamos a encontrar en estas dos obras es una descripción de la Inglaterra de los ochenta y de una sociedad que necesita un cambio. Churchill nos habla, por ejemplo, de las dificultades por las que la mujer ha tenido que pasar en su intento de cambiar la sociedad, haciendo frente a numerosos y diferentes obstáculos impuestos por un entorno en el que el capitalismo y el patriarcado triunfan, y en el que solamente pueden vivir aquellos que tienen suficiente poder para lograr el éxito a través de la tiranía, la división y la opresión.

Odisea, $n^{\circ} 2,2002$ 
En Serious Money se aprecia su respuesta a los escándalos financieros de los ochenta. La obra se escribió precisamente cuando la actividad en la bolsa alcanzaba su punto más álgido con el fenómeno del Big Bang de 1986: "[The Big Bang] involved the deregulation of the City and the opening of its doors to outsiders who seemed to threaten to dismantle traditional hierarchies of stockbroking and to overturn entrenched class assumptions" (Waugh, 1995:19).

La trama refleja el comercio oscuro de valores de bolsa que eran ilegalmente manipulados mediante una maniobra de adquisición, así como la desviación de la ayuda extranjera a los "bolsillos privados".

Churchill nos presenta a gente de negocios llena de avaricia y egoísmo. Este tipo de gente lo encontramos a lo largo de toda la obra con un objetivo único: el enriquecimiento propio. Son descritos como hombres y mujeres que triunfan, no haciéndose distinción de sexo, puesto que si tienen la capacidad para aportar beneficios a esta sociedad industrial y financiera, son bienvenidos a este mundo donde no hay ni lugar ni tiempo para pensamiento humano alguno. Pasemos pues a examinar los personajes más característicos.

Scilla Todd, es uno de los mejores ejemplos para mostrar el tipo de gente que la obra presenta. Scilla es realista, individualista, egoísta, fuerte y sobre todo avariciosa; cumple, por tanto, los principales requisitos necesarios para sobrevivir en la sociedad capitalista. Tiene un hermano, Jake, que representa la víctima del sistema; dedica toda su vida a los negocios arriesgando demasiado en sus tratos. Sin embargo, cuando Jake desaparece, nada cambia. Sus compañeros - incluso su hermana Scilla - permanecen indiferentes. Al principio parece muy afectada, pero esta conmoción desaparecerá más adelante, dejándose ver su verdadera naturaleza, puesto que lo único que le interesa no es la preocupación por la muerte del hermano sino la búsqueda de los negocios de éste, así como de su fortuna para apoderarse de ella. Todos forman parte de un mundo despreocupado y materialista, en el que no es difícil constatar la falta de sensibilidad y solidaridad de los personajes. Como Corman, un miembro de la compañía, dice, "hay una prioridad de trato sobre el asunto de Jake", la cual queda patente en el siguiente diálogo:

NIGEL.

Mr Corman, I'm deeply shocked that anyone associated with your company should be touched by the slightest breath / of scandal.

CORMAN.

The deal's in no way affected by his death.

( The deal is the priority.)

(1.217)

Grimes representa otra característica esencial de todo hombre de negocios: la agresividad, un valor indispensable si se quiere tener éxito en el mundo financiero. Grimes se refiere a esta agresividad cuando dice :

My school reports used to say I was too aggressive

(but it's come in quite useful).

My old headmaster wouldn't call me a fool again.

I got a transfer fee like a footballer. He thought I was a hooligan.

$\mathrm{He}$ goes, you fool boy, you're never going to get to work,

What use is a CSE in metalwork?

I could kiss his boots the day he kicked me out of school. 
Todos estos valores de agresividad, avaricia, individualidad y egoísmo son indispensables para la ideología thatcherista del éxito. El siguiente diálogo ilustra esta filosofía:

\section{DURKFELD.}

I do OK, sure, I'm not talking greed.

I'm talking how I mean to succeed.

(My father came to this country - forget it.)

Which of us does this company need?

I'm talking indispensable.

MERRISON.

And my father? You think I'm some kind of patrician?

I was sweeping floors in my uncle's delicatessen

So don't -

The company needs us both. Be sensible.

There's two aspects to the institution.

Nobody means to imply they underestimate your invaluable contribution.

I need to understand what you're saying here so let's set a time we can have a further talk.

DURKFELD.

You don't seem to get it. You're sitting in my chair. Walk.

(1.210)

Esta escena refleja la ruptura de dos socios codirectores de la empresa Klein Merrick, el comerciante Durkfeld y el banquero Merrison. Durkfeld es un negociante cansado de "hacer dinero" para los demás, y tiene una idea fija consistente en la posibilidad de dedicarse a los negocios en solitario. Merrison no logra entender la posición expuesta por su socio e intenta que reconsidere su decisión, mostrándole que su contribución es "invaluable". Sin embargo, la decisión tomada por Durkfeld es inapelable, viéndose así cómo el individualismo triunfa por encima del bien común. Él representa el modelo individualista en el mundo laboral para producir beneficio propio, y su actitud está acorde con la filosofía impulsada desde el gobierno, como apunta Samuel Brittan (1991:2): "The sources of economic growth do not lie with government. The Thatcher administration, did, however, do something to create the conditions in which Britain could start to catch up with its partners and competitors".

La política dirigida a eliminar las regulaciones y obstáculos burocráticos que encontraban las empresas, por un lado, se utilizó como medida para conseguir el auge económico. Pero, por otro lado, tuvo como consecuencia la falta de solidaridad del mundo empresarial en su afán de obtener más ganancias. Por tanto, estamos ante una nueva situación que Zac, otro banquero asociado con Klein Merrick, predice:

The financial world won't be the same again

Because the traders are coming down the fast lane. 
They don't even know it themselves, they're into fucking or getting a Porsche, getting a Porsche and a Mercedes Benz. $(1.210)$

Zac habla de la nueva expansión de negocios corruptos. Describe esta nueva situación de los negociantes y los banqueros, comparando el entramado de los negocios financieros con la selección natural descrita por Darwin: "It's like Darwin says, survival of the fit" (1.210). Nos presenta un mundo corrupto y violento, una jungla en la que puede sobrevivir el individuo con la capacidad adecuada, es decir, el individuo conocedor del sistema y lo suficientemente poderoso para avanzar en él.

Otra forma interesante de ilustrar este desarrollo corrupto es describiéndolo como un "juego". Al igual que en la teoría darwinista, el más fuerte gana y solamente el más poderoso y audaz llega a triunfar. El personaje que mejor personifica este juego es Frosby, el cual nos introduce en el mundo de la bolsa. Cada jugador tiene que dar lo mejor de sí en el menor tiempo posible, puesto que participa en una carrera interminable, en la que no hay tiempo para el descanso. En la sociedad competitiva no existe el compañerismo, sino el egoísmo, la hipocresía y la envidia, siendo ésta última una característica representada por Frosby, que tiene una vida vacía y solitaria. Su rencor es tan extremo que no duda en llamar al DTI (Department of Trade and Industry) para que investiguen al envidiado Jake.

Inevitablemente, el factor "riesgo" siempre acompaña al juego. Es uno de los valores que conforman la política económica thatcherista, como menciona el banquero Merrison en las siguientes palabras:

Partners should be willing to risk their own capital.

I told them, man is a gambling animal.

Risk is one of our company traditions.

Old Benny Klein took risks, the latest news

Meant profit, they'd say on Wall Street 'Let the Jews

Have that one', and he would.

(1.208)

Tomar decisiones arriesgadas era un requisito para un crecimiento económico a corto plazo. A la vez, estas decisiones podrían desembocar en deudas, aunque este asunto no causaba preocupación alguna entre los hombres de negocios, como se puede ver en Corman cuando dice que "...being in debt is the best way to be rich."(1.229).

Es en el segundo acto donde Churchill describe el lado mas inhumano de la sociedad, mostrando así la tiranía del poder. En la siguientes palabras de Zac podemos apreciar esta tiranía:

The so-called third world doesn't want our charity or aid.

All they need is the chance to sit down in front of some green

screens and trade.

(They don't have the money, sure, but just so long as they have freedom from communism so they can do it when they do have the money.) 
El mensaje que se desprende de este párrafo es que Inglaterra pertenece a los países desarrollados, y que dichos países no están interesados en ayudar a los países subdesarrollados a conseguir la independencia económica, puesto que significaría perder el control absoluto del mercado.

Es al final del segundo acto, con la conversación entre Corman y Gleason, un ministro del Gabinete, cuando hay un ataque directo al gobierno:

\section{CORMAN.}

You'd stop the scandal breaking anyway.

Are you telling me you can't control the press?

GLEASON.

Yes, but we'd break you. Do you want to try?

You drop the bid. We stop the DTI.

CORMAN.

Why pick on me ? Everyone's the same.

I'm just good at playing a rough game.

GLEASON.

Exactly, and the game must be protected.

You can go on playing after we're elected.

Five more glorious years free enterprise,

And your services to industry will be recognised.

CORMAN.

Cunt. Right. Good.

At least a knighthood.

(2.299)

En este diálogo se revela el lado más oscuro de la política conservadora. Se critica la inminente victoria de los Tories en las elecciones de 1987 anunciándose, por tanto, cinco años más de corrupción y avaricia. La cuestión aquí tratada refleja la importancia de dar una buena imagen política y, sobre todo, en el período preelectoral. Churchill pone de manifiesto que no es lo mismo lo que se promete en las elecciones que lo que se hace realmente una vez ganadas éstas, por lo que los intereses de los ciudadanos pasan a un segundo plano.

Por tanto, una vez más, Churchill nos ilustra la naturaleza superficial y avariciosa de los que sólo quieren conseguir más poder y más riqueza. Viven en una continua corrupción que cualquier negociante lleva a cabo, como Scilla, cuando ella misma reconoce su naturaleza en afirmaciones como: "I'm greedy and completely amoral / I've the cunning and connection of the middle class / And I'm tough as a yob" (2.305).

Este sentimiento de avaricia queda igualmente manifiesto en Top Girls. Aquí, Marlene, recién ascendida al cargo de directora de la agencia de empleo TOP GIRLS, representa claramente ese afán por conseguir el éxito en la sociedad capitalista. Como Joseph Marohl (1987:379) indica: "work, promotion, money, and success are topics of conversation among the characters throughout the play". Si en Serious Money, Churchill denuncia el sistema por medio de la descripción de los hombres de negocios y de los banqueros, inmersos todos ellos en una atmósfera dinámica de trabajo, en Top Girls la peculiaridad consiste en atacar al sistema capitalista y al individualismo desde una perspectiva feminista. Churchill hace una descripción de la vida contemporánea en Inglaterra, respondiendo por un lado y de forma general al sistema preconizado durante la época thatcherista . Por otro, critica a la señora Thatcher a través de Marlene, personaje que cumple todos los requisitos para ser nombrada

Odisea, $n^{\circ} 2,2002$ 
"heroína thatcherista". Por eso, primero vamos a adentrarnos en la obra desde un punto de vista más contextual para luego centrarnos en la señora Thatcher.

La historia comienza en un restaurante donde Marlene celebra su reciente ascenso a directora con un grupo de amigas, entre las que se mezclan personajes históricos y ficticios: Isabella Bird, una viajera escocesa del siglo XIX; Lady Nijo, una monja japonesa del siglo XIII que había sido antes cortesana; Dull Gret, la imagen de un cuadro de Bruegel; Pope Joan, una legendaria niña prodigio que con tan solo doce años de edad abandonó su casa y que posteriormente, haciéndose pasar por un varón, llegó a Papa; y, por último, Griselda la paciente, un personaje de la obra de Chaucer, The Clerk's Tale, que representa a la esposa obediente, siempre fiel y sumisa a su marido.

Aunque cada una de estas mujeres relata su vida de forma individual, todas ellas comparten una experiencia común: la pérdida de control de sus vidas debido a la opresión a la que han sido sometidas. Churchill nos aproxima a cada una de las historias contadas a través del conocido "overlapping dialogue": los diálogos llegan a solaparse con el propósito de hacer las experiencias contadas por cada una de ellas compartidas y vividas por todas. Por tanto, estamos ante una obra en la que la mujer se encuentra sometida al poder de una sociedad capitalista basada en el individualismo y en el sistema patriarcal.

Griselda representa la condición de la mujer en la sociedad siendo el mejor ejemplo de los efectos del patriarcado: permanece ciegamente sumisa, pasiva y obediente hasta el punto de anteponer su marido a sus propios hijos ya que, según Griselda, "a wife must obey her husband / And of course I must obey the Marquis" (1.75).

El amor y la devoción a su marido es tal que no llega a reconocer el daño ocasionado, defendiéndolo de todas sus acciones y decisiones tomadas, y eximiéndolo de toda responsabilidad de su sufrimiento: ("He suffered so much all those years.") (1.79). En vez de expresar su propio sentimiento y su dolor, solamente tiene cabida en su mente la preocupación por su marido.

Isabella es otro ejemplo más de esta opresión varonil: "I tried to do what my father wanted"(1.57). / "Yes , I forgot all my Latin. But my father was the mainspring of my life and when he died I was so grieved" (1.58).

Sobre este asunto, Linda Fitzsimmons (1987: 26-27) argumenta que "family inheritance is part of the system of oppression (...) in both these plays, then, Churchill presents the notion of motherhood and feminity as constructed rather than naturally, shows women as systematically oppressed by both capitalism and patriarchy". Pero en Top Girls Churchill deplora que la alternativa a esta opresión sea el matriarcado: "The action indicates that the female perspective is capable too of drawing class distinctions and enforcing a patriarchlike matriarchy based on tyranny and division" (Marohl, 1987: 381).

Todas estas "new women" disponen de la capacidad y la fuerza necesarias para conseguir triunfar en la vida. Son virtudes exaltadas por la orgullosa promotora de esta nueva etapa de la mujer, Marlene, y que expresa con gran euforia cuando dice: "We've all come a long way. To our courage and the way we change our lives and our extraordinary achievements" (1.67). Estas mujeres representan el feminismo burgués que consiste en "accepting society as it is, and focussing only on the question of how individual women can succeed within it" (Fitzsimmons, 1987: 19).

Son mujeres con gran ambición que se muestran orgullosas de sus éxitos personales en el mundo laboral. Este afán de prosperidad en ningún momento desafía al sistema, y por tanto, no aporta solución alguna. Por el contrario, con esta actitud, lo único que se consigue es el refuerzo de la filosofía individualista en la que no cabe el cambio social.

Su afán de superación se puede observar en Win, una empleada de la agencia Top Girls cuando dice: "men are awful bullshitters, they like to make out jobs harder than they are. 
Any job I ever did I started doing it better than the rest of the crowd and they didn't like it". (2.3.119). Este concepto feminista es el que Churchill critica, puesto que el triunfo individual de la mujer no acaba con la hegemonía de poder y con la tiranía del sistema. De igual modo, tampoco debe entenderse como una oposición binaria entre masculinidad y feminidad, sino que habría que hablar de una relación de "opresor" y "oprimido":

The play in performance moves the audience from the apparent dichotomy of "female/male", which Marlene's discourse asserts, to the underlying dichotomy of "oppresor/ oppresed" which is the effect of phallocentric hierarchism and which operates outside of the classification of sex and gender. (Marohl, 1987:387)

Pasemos ahora a ocuparnos de Marlene, ya que representa a la heroína thacherista por excelencia. Merece ser analizada la analogía existente entre Marlene y Margaret Thatcher. Al igual que ella, Marlene proviene de una familia de clase media baja, pero reniega de su origen, ya que implica un obstáculo para alcanzar sus propios intereses. El hecho de abandonar a su hija por su incorporación, y su consiguiente triunfo, en el mundo laboral, nos muestra la prioridad de su trabajo sobre su maternidad, prioridad vista anteriormente en Serious Money.

Pero es en el acto tercero donde aparece la crítica a la ideología conservadora thatcherista. Este acto revela aspectos de la vida de Marlene que merecen ser mencionados. Ella no sólo abandonó su hogar sino que renegó de su maternidad (otorgando el papel de madre a su hermana Joyce), demostrando así su alienación con respecto a su familia y a su responsabilidad familiar:

MARLENE. I don't mean anything personal. I don't believe in class. Anyone can do anything if they've got what it takes.

JOYCE. And if they haven't?

MARLENE. If they're stupid or lazy or frightened, I'm not going to help them get a job, why should I?

JOYCE. What about Angie?

MARLENE. What about Angie?

JOYCE. She's stupid, lazy and frightened, so what about her?

MARLENE. You run her down too much. She'll be all right.

JOYCE. I don't expect so, no. I expect her children will say what a wasted life she had. If she has children. Because nothing's changed and it won't with them in.

En primer lugar, tratemos de entender bien cuáles son las posturas ideológicas que siguen Marlene y su hermana Joyce. Marlene adopta una postura totalmente individualista que refleja su apoyo a la política conservadora de la época. Su argumento a favor del conservadurismo, además, no se cuestiona y se basa en mensajes prefijados, como apunta Anne Varty (1994:79):

Her connotations of 'working class' are those clichés given by the tabloid press, notoriously male-run and usually Tory in allegiance. Her subsequent protestation 'I want to be free in a free world' reflects the extent to which she regurgitates the 
clichés of Thatcherism, when her professional success (managing director of an employment agency for women) depends on putting price tags on the most insubstantial qualities, and the certainty that nothing is free.

Por el contrario, Joyce defiende a la clase social trabajadora, que incluye a toda persona que sea "stupid or lazy or frightened". El hecho de adoptar a Angie es una prueba de ello.

En segundo lugar, debe ser mencionada la relación de Marlene y su hija Angie. La relación de madre/hija ha sido objeto de estudio en otras obras de Churchill, siendo Fen (1983) una de las más representativas: "In such relationships, the mother, by definition, represents the memories and traditions of the past and the realities of the present, while the daughter, by definition, represents the possibilities of the future" (Swanson, 1986: 49).

En este caso concreto, podemos ver que las posibilidades de futuro de Angie no son esperanzadoras, debido a que no ha recibido ni amor maternal ni educación alguna. Marlene no está dispuesta a sacrificar su éxito por ayudar a Angie: "Marlene embodies the characteristics of the popular myth of career woman as castrating female and barren mother" (Marohl, 1987: 377).

Marlene, por tanto, representa el fracaso, puesto que su hija es el producto de su abandono como madre. Este fracaso se puede interpretar como el fracaso del Thatcherismo como sistema político y como sistema que gobierna las relaciones personales. Podemos afirmar, pues, que Marlene pierde toda heroicidad, ya que es un mero producto de la sociedad económica thatcherista.

En la última imagen de la obra vemos a Angie despertando de una pesadilla y refiriéndose a ella como "frightening". Esta palabra es usada también en Serious Money por Frosby (1.216), el empleado solitario de la agencia Liffe. En ambas obras vemos un sentimiento común, el miedo que presentan los personajes a un futuro incierto. Ante esta inquietante incertidumbre, se hace patente la necesidad de un modelo de cambio social, y Churchill pone todo su empeño en subrayar esta exigencia, como ha señalado Cristopher Innes (1992:171): "in manipulating time and stage conventions, as well as sexual stereotypes, her work undermines recieved notions of personality, and challenges the way we perceive reality (...) the theatre itself is affirmed as the model for positive change". El sistema thatcherista presentado en Serious Money y Top Girls debe ser reemplazado, puesto que, según hemos visto, es un sistema en el que la posición del ser humano es insostenible.

\section{BIBLIOGRAFÍA}

Brittan, S. 1991 (1989). "The Thatcher government's economic policy”. The Thatcher Effect. Eds. D. Kavanagh and A. Seldon. 1-37.

Churchill, C. 1996 (1990). Churchill: Plays Two: Top Girls. London: Methuen. 51-141. 1996 (1990). Churchill: Plays Two: Serious Money. London: Methuen. 194309.

1996 (1990). Churchill: Plays Two: Fen. London : Methuen. 144-192.

Fernández Sánchez, J.F. 1999. El Thatcherismo. Historia y Análisis de una Época. Universidad de Almería: Servicio de Publicaciones.

Fitzsimmons, L. 1987. "I won't turn back for you or anyone: Caryl Churchill socialistfeminist theatre". Essays in Theatre 1, 6: 19-29. 
Innes, C. 1992. Modern British Drama (1890-1990).Cambridge: C.U.P.

Marohl, J. 1987. "De-realised women: performance and identity in Top Girls". Modern Drama 3, 30: 376-388.

O'Shaugnessy, M. 1996. “The lady turns back: the thatcherite discourse on thatcherism." Atlantis XVIII, 1-2: 295-305.

Swanson, M. 1986. "Mother/daughter relationships in three plays by Caryl Churchill". Theatre Studies 31. 49-66

Thomas, J. 1992. "The plays of Caryl Churchill: essays in refusal". The Death of the Playwright? Ed. A. Page. Basingstone: Macmillan. 160-185.

Varty, A. 1994. "From queens to convicts: status, sex and language in contemporany British women's drama". Essays and Studies 47: 65-89.

Waugh, P. Harvest of the Sixties. 1995. Oxford: O.U.P. 